\title{
SHARPLY TWO-TRANSITIVE FAMILIES OF PERMUTATIONS ON AN IMMUNE SET
}

\author{
J. C. E. DEKKER
}

(Received 4 March 1977)

Communicated by J. N. Crossley

\begin{abstract}
It is known that there is a finite affine (or projective) plane of order $n$ if and only if there is a sharply two-transitive set of permutations of degree $n$. This paper deals with a generalization of this theorem, in which finite sets are replaced by isolated sets, cardinalities by isols and certain effectiveness conditions are imposed on the two systems involved which are trivially satisfied in the finite case.
\end{abstract}

Subject classification (Amer. Math. Soc. (MOS) 1970): primary 02F40, secondary 05B25.

\section{Introduction}

We use the word "family" for a collection of permutations on some set; a family is therefore not necessarily a group under composition. Let $\mathscr{P}$ be a family of permutations on some set $S$ of cardinality $\geqq 2$. Then $\mathscr{P}$ is sharply two-transitive (STT), if

(a) $\left\{\begin{array}{l}\text { for } a, b, c, d \in S, a \neq c, b \neq d, \mathscr{P} \text { has exactly one member } f \text { such that } \\ f(a)=b \text { and } f(c)=d \text {. }\end{array}\right.$ In the special case that $S$ is finite, (a) implies

(b) $\left\{\begin{array}{l}\text { for } a, b \in S, f \in \mathscr{P}, f(a) \neq b, \mathscr{P} \text { has exactly one member } g \text { such that } \\ g(a)=b \text { and } g(x) \neq f(x) \text {, for all } x \in S,\end{array}\right.$ but this is no longer true if $S$ is infinite, Hall (1943). It is known (Hall (1943), Dembowski (1968), p. 140) that for $n \geqq 2$,

(c) $\{$ there is an affine (or projective) plane $\Gamma$ of order $n$ if and only if there is an STT family $\mathscr{P}$ of permutations on some finite set of cardinality $n$.

It is the purpose of this paper to generalize (c) to the case where $S$ is an isolated set of nonnegative integers and certain effectiveness conditions are imposed on the affine plane $\Gamma$ and the STT family $\mathscr{P}$. 


\section{Preliminaries}

The word "number" will be used for nonnegative integer, "set" for collection of numbers, the letter $\varepsilon$ for the set of all numbers, $F$ for the class of all r.e. sets and $Q$ for the class of all finite sets. If $f$ is a mapping from some subcollection of $\varepsilon^{n}$ into $\varepsilon$, we write $\delta f$ for the domain of $f$ and $\rho f$ for the range of $f$. Effectiveness will be expressed in terms of the existence of certain partial recursive functions. We shall assume the reader to be familiar with the basic terminology concerning RETs, that is recursive equivalence types, and their elementary properties, Dekker and Myhill (1960). We often use the proposition that the sets $\alpha$ and $\beta$ are recursively equivalent [written: $\alpha \simeq \beta$ ] if and only if there is a one-to-one function $f$ from $\alpha$ onto $\beta$ such that both $f$ and $f^{-1}$ have partial recursive extensions.

All affine planes and STT families to be considered will be countable. We define an affine plane as an ordered triple $\Gamma=\langle\delta, \nu$, inc. $\rangle$, where $\delta$ (the set of all points) and $\nu$ (the set of all lines) are disjoint sets, $\delta \cup \nu$ has an infinite complement (to permit completion to a projective plane without getting outside $\varepsilon$ ), inc. is a subcollection of $\delta \times \nu$ and the three classical axioms hold. The lines $l$ and $m$ of $\Gamma$ are parallel, if $l=m$ or $l$ and $m$ have no point in common. The parallel relation [written: II] defines a partition of $\nu$; the members of this partition are the parallel sets of $\Gamma$. Every affine plane has at least three parallel sets. Let $a, b \in \delta, a \neq b, l, m \in \nu$, not $(l \| m)$. Then we write $a \cdot b$ for the line joining $a$ and $b$, and $l \cap m$ for the point in which $l$ and $m$ intersect. Let $p \in \delta, l \in \nu$. Then $\delta_{l} \doteq\{x \in \boldsymbol{\delta} \mid x$ inc. $l\}$. If $\alpha$ is any set we write $(\alpha \times \alpha)^{-}$for $\{(x, y) \in \alpha \times \alpha \mid x \neq y\}$; in the special case that $\alpha \subset \nu$, $(\alpha \times \alpha)^{-}=\{(x, y) \in \alpha \times \alpha \mid \operatorname{not}(x \| y)\}$. With $\Gamma$ we associate the functions $L_{\Gamma}$, $P_{\Gamma}, Q_{\Gamma}$ :

$$
\begin{aligned}
\delta L_{\Gamma} & =(\delta \times \delta)^{-}, \quad L_{\mathrm{\Gamma}}(a, b)=a \cdot b, \\
\delta P_{\Gamma} & =(\nu \times \nu)^{-}, \quad P_{\Gamma}(l, m)=l \cap m, \\
\delta Q_{\Gamma} & =\delta \times \nu, \quad Q_{\mathrm{\Gamma}}(p, l)=\text { line through } p \text { which is } \| l .
\end{aligned}
$$

Definition D1. The affine plane $\Gamma=\langle\delta, \nu$, inc. $\rangle$ is an affine $\omega$-plane, if

(1) there are three mutually disjoint r.e. sets $\bar{\delta}, \bar{\nu}, \bar{\tau}$ such that $\delta \subset \bar{\delta}, \nu \subset \bar{\nu}$ and $\bar{\tau}$ is infinite.

(2) the functions $L_{\Gamma}, P_{\Gamma}, Q_{\Gamma}$ have partial recursive extensions.

Affine $\omega$-planes were introduced in (Dekker, 1977), where the following propositions were proved. If $l$ and $m$ are lines of an affine $\omega$-plane $\Gamma=\langle\delta, \nu$, inc. $\rangle$, then $\delta_{l} \simeq \delta_{m}$. Call $N=\operatorname{Req} \delta_{t}$ the order of $\Gamma$ [written: $o[\Gamma]$. $\Gamma$ is called isolic (immune), if $N$ is an isol (infinite isol) or equivalently, if $\delta$ and $\nu$ are isolated (immune) sets. We have 
(d) $\operatorname{Req} \delta=N^{2}$, Req $\nu=N^{2}+N$, every parallel set of $\Gamma$ has $R E T N$, for an arbitrary affine $\omega$-plane $\Gamma=\langle\delta, \nu$, inc. $\rangle$ of order $N$. Moreover, writing $c=2^{\boldsymbol{\kappa}_{0}}$,

(e) $\left\{\begin{array}{l}\text { there are exactly } c \text { affine } \omega \text {-planes; among these there are } c \text { mutually } \\ \text { nonisomorphic immune ones. }\end{array}\right.$

DEFINITION D2. An $\omega$-STT family of permutations is an ordered triple $\mathscr{P}=\langle\tau, \sigma, p\rangle$, consisting of a nonempty set $\tau$, a set $\sigma$ of cardinality $\geqq 2$ and a function $p$ from $\tau \times \sigma$ into $\sigma$ such that:

(1) $p$ has a partial recursive extension and for each $t \in \tau, p(t, x)$ is a permutation of $\sigma$,

(2) given any elements $a, b, c, d \in \sigma$ with $a \neq c, b \neq d$, there is a unique $t \in \tau$ such that $p(t, a)=b, p(t, c)=d$ and this $t$ can be effectively found.

Remarks R1. We often write $p_{t}(x)$ instead of $p(t, x)$, in particular if we wish to keep $t$ temporarily fixed. Thus $\mathscr{P}=\left\{p_{t} \mid t \in \tau\right\}$; we call $\tau$ the index set of $\mathscr{P}$ and $\sigma$ the base set of $\mathscr{P}$. In view of (2), the mapping $t \rightarrow p_{t}$ from $\tau$ into the family of all permutations of $\sigma$ is one-to-one.

R2. Condition (2) can be made more precise: there is a function $q(a, b, c, d)$ with a partial recursive extension such that

$$
\delta q=\left\{(a, b, c, d) \in \sigma^{4} \mid a \neq c \& b \neq d\right\}, \quad \rho q \subset \tau,
$$

and for $(a, b, c, d) \in \delta q$, the number $t \in \tau$ with the desired properties equals $q(a, b, c, d)$.

R3. Let $\mathscr{P} \cdot=\langle\tau, \sigma, p\rangle$ be an $\omega$-STT family (of permutations) on a set $\sigma$. Suppose that $\tau \simeq \tau^{*}$ and that $q(x)$ is a partial recursive one-to-one function with $\tau \subset \delta q, q(\tau)=\tau^{*}$. Put $\mathscr{P}^{*}=\left\langle\tau^{*}, \sigma, p^{*}\right\rangle$, where

$$
p^{*}\left(t^{*}, x\right)=p\left[q^{-1}\left(t^{*}\right), x\right], \quad \text { for } t^{*} \in \tau^{*}, x \in \sigma .
$$

Then $\mathscr{P}^{*}$ is also an $\omega$-STT family. This shows that only Req $\tau$ is relevant for the existence of a function $p$ such that $\langle\tau, \sigma, p\rangle$ is an $\omega$-STT family on $\sigma$. We therefore say that there exists an $\omega$-STT family on a set $\sigma$, if there is an ordered pair $\langle\tau, p\rangle$ such that $\langle\tau, \sigma, p\rangle$ is an $\omega$-STT family on the set $\sigma$.

\section{Eight Propositions}

Let $\sigma$ be a finite set of cardinality $\geqq 2$. Then there exists an $\omega$-STT family on $\sigma$ if and only if there exists an STT family on $\sigma$. Moreover, the existence of an STT family on $\sigma$ clearly depends only on the cardinality of $\sigma$. This suggests the following recursive analogue. 
Proposition C1. Whether there exists an $\omega$-STT family of permutations on the set $\sigma$ depends only on $\operatorname{Req} \sigma$. In other words, if $\sigma \simeq \sigma^{*}$, there exists an $\omega$-STT family on $\sigma$ if and only if there exists an $\omega$-STT family on $\sigma^{*}$.

Proof. Let $\mathscr{P}=\langle\tau, \sigma, p\rangle$ be an $\omega$-STT family on $\sigma$. Assume $\sigma \simeq \sigma^{*}$, say $\sigma \subset \dot{\delta f}, f(\sigma)=\sigma^{*}$, for some partial recursive one-to-one function $f$. Put $p^{*}(t, x)=f p\left[t, f^{-1}(x)\right]$, for $t \in \tau, x \in \sigma^{*}$, then $\mathscr{P}^{*}=\left\langle\tau, \sigma^{*}, p^{*}\right\rangle$ is an $\omega$-STT family on $\sigma^{*}$.

Definition D3. Let $\mathscr{P}=\langle\tau, \sigma, p\rangle$ be an $\omega$-STT family on $\sigma$. Then Req $\tau$ is the order of $\mathscr{P}$ [written: $o(\mathscr{P})$ ] and Req $\sigma$ is the degree of $\mathscr{P}$ [written: $d(\mathscr{P})]$.

Proposition C2. Let $\mathscr{P}=\langle\tau, \sigma, p\rangle$ be an $\omega$-STT family on $\sigma$. Then $\tau$ is isolated if and only if $\sigma$ is isolated, that is, $o(\mathscr{P})$ is an isol if and only if $d(\mathscr{P})$ is an isol.

Proof. We shall show that

(*) $\tau$ has a subset in $F-Q \Leftrightarrow \sigma$ has a subset in $F-Q$.

For suppose $t_{n}$ is a one-to-one recursive function with $\rho t \subset \tau$. Let $a$ and $b$ be distinct elements of $\sigma, c_{n}=p\left(t_{n}, a\right), d_{n}=p\left(t_{n}, b\right)$, for $n \in \varepsilon$. Then $c_{n}$ and $d_{n}$ are recursive functions with $\rho c, \rho d \subset \sigma$. Note that $i \neq k$ implies $\left\langle c_{i}, d_{i}\right\rangle \neq\left\langle c_{k}, d_{k}\right\rangle$. Hence either $\rho c$ or $\rho d$ is an infinite r.e. subset of $\sigma$. Now suppose that $s_{n}$ is a one-to-one recursive function with $\rho s \subset \sigma$. Put $a=s_{0}$, $b=s_{1}, c=s_{2}, d_{n}=s_{n+3}$, for $n \in \varepsilon$; then $a \neq c, b \neq d_{n}$, for $n \in \varepsilon$; hence there is a function $r_{n}$ from $\varepsilon$ into $\tau$ such that $p\left(r_{n}, a\right)=b, p\left(r_{n}, c\right)=d_{n}$. The function $r_{n}$ is one-to-one and recursive; hence its range is an infinite r.e. subset of $\tau$.

Definition D4. An $\omega$-STT family $\mathscr{P}=\langle\tau, \sigma, p\rangle$ is isolic if the sets $\tau$ and $\sigma$ are isolated.

Proposition C3. Let $\mathscr{P}=\langle\tau, \sigma, p\rangle$ be an isolic $\omega$-STT family of degree $N$. Suppose that $a, b \in \sigma$ (where $a=b$ or $a \neq b$ ) and $\tau_{a b}=\{t \in \tau \mid p(t, a)=b\}$. Then $\mathscr{P}$ has $N-1$ members which map a onto $b$, that is, $\operatorname{Req} \tau_{a b}=N-1$.

Proof. Assume the hypothesis. Let $c \in \sigma, c \neq a$. Henceforth we keep $a$, $b, c$ fixed. Define a function $f_{b}$ by: $\delta f_{b}=\sigma-(b)$ and for $x \in \delta f_{b}$,

$$
f_{b}(x)=\text { unique } t \in \tau \text { with } p_{t}(a)=b \text { and } p_{t}(c)=x .
$$

Clearly, $f_{b}$ maps $\sigma-(b)$ one-to-one into $\tau_{a b}$ and has a partial recursive extension. Let $t^{\prime} \in \tau_{a b}$. Put $x^{\prime}=p_{t^{\prime}}(c)$; then $x^{\prime}$ can be computed from $t^{\prime}$; also, $f_{b}\left(x^{\prime}\right)=t^{\prime}$. Thus $f_{b}$ maps $\sigma-(b)$ onto $\tau_{a b}$ and $f_{b}^{-1}$ has a partial recursive extension. It follows that $\sigma-(b) \simeq \tau_{a b}$, i.e., that $\operatorname{Req} \tau_{a b}=\operatorname{Req} \sigma-1=N-1$. 
REMARK R4. Note that given two elements $b, x \in \sigma$ with $b \neq x$, the element $f_{b}(x)$ can be computed.

Proposition C4. Every isolic $\omega$-STT family of degree $N$ has order $N(N-1)$.

Proof. Let $\mathscr{P}=\langle\tau, \sigma, p\rangle$ be an isolic $\omega$-STT family and let $a, c$ be distinct elements of $\sigma$. In this proof we keep $a$ and $c$ fixed. Define a function $g$ by:

$$
\delta g=\{j(b, x) \in \varepsilon \mid b, x \in \sigma \& b \neq x\}, \quad g j(b, x)=f_{b}(x),
$$

where $f_{b}(x)$ is the function defined in the proof of C3. In view of R4 the function $g$ has a partial recursive extension. Let $j(b, x), j\left(b^{\prime}, x^{\prime}\right) \in \delta g$ and $j(b, x) \neq j\left(b^{\prime}, x^{\prime}\right)$. If $b \neq b^{\prime}, \tau_{a b}$ and $\tau_{a b^{\prime}}$ are disjoint; hence $f_{b}(x) \in \tau_{a b}$ and $f_{b^{\prime}}\left(x^{\prime}\right) \in \tau_{a b^{\prime}}$ imply $f_{b}(x) \neq f_{b^{\prime}}\left(x^{\prime}\right)$. If on the other hand $b=b^{\prime}$, we have $x \neq x^{\prime}$ and $f_{b}(x) \neq f_{b} \cdot\left(x^{\prime}\right)$ since $f_{b}$ is one-to-one. We have shown that $g$ is one-to-one. Since $\tau=\cup\left\{\tau_{a b} \mid b \in \tau\right\}$, while $f_{b}$ maps $\sigma-(b)$ onto $\tau_{a b}$, for $b \in \tau$, we see that $\rho g=\tau$. Assume $t \in \tau$; then $g j(b, x)=t$, for $b=p_{t}(a)$ and $x=p_{t}(c)$. Hence $g^{-1}(t)$ can be computed from $t$, and $g^{-1}$ has a partial recursive extension. Thus $\tau \simeq \delta g$ and

$$
o(\mathscr{P})=\operatorname{Req} \tau=\operatorname{Req} \delta g=\operatorname{Req} j\left[(\sigma \times \sigma)^{-}\right]=N^{2}-N=N(N-1) .
$$

Corollary. If $\mathscr{P}=\langle\tau, \sigma, p\rangle$ is an isolic $\omega$-STT family, the set $\sigma$ is infinite if and only if the set $\tau$ is infinite, that is, $\sigma$ is immune if and only if $\tau$ is immune.

Definition D5. An $\omega$-STT family $\mathscr{P}=\langle\tau, \sigma, p\rangle$ is finite (immune), if the sets $\tau$ and $\sigma$ are finite (immune).

Definition D6. An $\omega$-STT family $\mathscr{P}=\langle\tau, \sigma, p\rangle$ is locally finite if for every two nonempty finite sets $\tau_{0}, \sigma_{0}$ with $\tau_{0} \subset \tau, \sigma_{0} \subset \sigma$, there exist finite sets $\tau^{*}, \sigma^{*}$ with $\tau_{0} \subset \tau^{*} \subset \tau, \sigma_{0} \subset \sigma^{*} \subset \sigma$ such that $\mathscr{P}^{*}=\left\langle\tau^{*}, \sigma^{*}, p^{*}\right\rangle$ with $p^{*}=$ $p \mid \tau^{*} \times \sigma^{*}$ is an $\omega$-STT family. Moreover, $\mathscr{P}$ is recursively locally finite if given the nonempty finite sets $\tau_{0}, \sigma_{0}$ such sets $\tau^{*}$ and $\sigma^{*}$ can be effectively found.

Proposition C5. Every isolic $\omega$-STT family is recursively locally finite.

Proof. We may assume without loss of generality that the sets $\sigma$ and $\tau$ of the $\omega$-STT family $\mathscr{P}=\langle\tau, \sigma, p\rangle$ are immune. Let nonempty finite sets $\tau_{0}, \sigma_{0}$ with $\tau_{0} \subset \tau, \sigma_{0} \subset \sigma$ be given. Suppose that $a, b, c, d$ are elements of $\sigma$ with $a \neq c, b \neq d$; from now on we keep these elements fixed. We may assume without loss of generality that $a, b, c, d \in \sigma_{0}$, for otherwise we adjoin them to $\sigma_{0}$. Put $p_{0}=p \mid \tau_{0} \times \sigma_{0}, \mathscr{P}_{0}=\left\langle\tau_{0}, \sigma_{0}, p_{0}\right\rangle$; then we can effectively test whether $\mathscr{P}_{0}$ 
is an $\omega$-STT family; if it is, we are done. Now suppose that $\mathscr{P}_{0}$ is not an $\omega$-STT family and that $q$ is the function mentioned in R2. We now adjoin (i) to $\sigma_{0}$ every element of the form $p_{t}\left(a_{1}\right)$, where $t \in \tau_{0}, a_{1} \in \sigma_{0}$, (ii) to $\tau_{0}$ any element of the form $q\left(a_{1}, a_{2}, a_{3}, a_{4}\right)$, where $\left(a_{1}, a_{2}, a_{3}, a_{4}\right)$ belongs to $\sigma_{0}^{4} \cap \delta q$. This yields finite sets $\tau_{1}, \sigma_{1}$ with $\tau_{0} \subset \tau_{1} \subset \tau, \sigma_{0} \subset \sigma_{1} \subset \sigma$. We continue this process until it terminates, that is, until we obtain finite sets $\tau_{k}, \sigma_{k}$ which are closed under the operations (i) and (ii). This will happen after finitely many steps, since the extension process is effective, while $\tau$ and $\sigma$ are immune. Let $\tau^{*}, \sigma^{*}$ be the finite sets obtained when the process terminates. Then $\mathscr{P}^{*}=$ $\left\langle\tau^{*}, \sigma^{*}, p^{*}\right\rangle$ with $p^{*}=p \mid \tau^{*} \times \sigma^{*}$ is a finite $\omega$-STT family such that $\sigma_{0} \subset \sigma^{*} \subset$ $\sigma, \tau_{0} \subset \tau^{*} \subset \tau$. Note that $\tau^{*}$ and $\sigma^{*}$ can be computed from $\tau_{0}, \sigma_{0}, p$ and $q$.

Proposition C6. Let $\mathscr{P}=\langle\tau, \sigma, p\rangle$ be an $\omega$-STT family. If $\mathscr{P}$ is isolic, it satisfies the following condition: given any elements $t \in \tau, a, b \in \sigma$ with $p(t, a) \neq b$, there is a unique $t^{\prime} \in \tau$ such that $p\left(t^{\prime}, a\right)=b$ and $p\left(t^{\prime}, x\right) \neq p(t, x)$, for all $x \in \sigma$, and this number $t^{\prime}$ can be effectively found from $t, a, b$.

Proof. Assume the hypothesis. Since (b) holds for a finite STT, we may suppose without loss of generality that $\tau$ and $\sigma$ are immune. Let $t \in \tau$, $a, b \in \sigma$ be given. Put $\tau_{0}=(t), \sigma_{0}=(a, b)$; then we can by C5 compute finite sets $\tau^{*}, \sigma^{*}$ such that $\tau_{0} \subset \tau^{*} \subset \tau, \sigma_{0} \subset \sigma^{*} \subset \sigma$ and $\mathscr{P}^{*}=\left\langle\tau^{*}, \sigma^{*}, p^{*}\right\rangle$ with $p^{*}=p \mid \tau^{*} \times \sigma^{*}$ is a finite $\omega$-STT family. Since $\mathscr{P}^{*}$ is finite, we can from $\tau^{*}, \sigma^{*}, p^{*}$ compute the unique $t^{\prime} \in \tau^{*}$ such that $p\left(t^{\prime}, a\right)=b$ and $p\left(t^{\prime}, x\right) \neq p(t, x)$, for all $x \in \sigma^{*}$. If we can show that $p\left(t^{\prime}, x\right) \neq p(t, x)$, for all $x \in \sigma-\sigma^{*}$, we are done. Let $\bar{x} \in \sigma-\sigma^{*}$; then there are finite sets $\bar{\tau}$ and $\bar{\sigma}$ such that $\tau^{*} \subset \bar{\tau} \subset \tau, \sigma^{*} \cup(\bar{x}) \subset \bar{\sigma} \subset \sigma$ and $\bar{P}=\langle\bar{\tau}, \bar{\sigma}, \bar{p}\rangle$ with $\bar{p}=p \mid \bar{\tau} \times \bar{\sigma}$ is a finite $\omega$-STT family. Let $\bar{t}$ be the unique element in $\bar{\tau}$ such that $p(\bar{t}, a)=b$ and $p(\bar{t}, x) \neq p(t, x)$, for all $x \in \bar{\sigma}$. Since $\sigma^{*} \subset \bar{\sigma}$ it follows that $\bar{t}=t^{\prime}$; hence $p\left(t^{\prime}, x\right) \neq p(t, x)$, for all $x \in \bar{\sigma}$, in particular, $p\left(t^{\prime}, \bar{x}\right) \neq p(t, \bar{x})$.

Proposition C7. Let $\mathscr{P}=\langle\tau, \sigma, p\rangle$ be an isolic $\omega$-STT family. Then there is an effective procedure which enables us, given $t \in \tau, s \in \sigma$, to find the unique solution in $\sigma$ of the equation $p_{t}(x)=s$.

Proof. Let $x_{0}$ be the desired solution. If the sets $\tau$ and $\sigma$ are finite, $x_{0}$ can be found by computing $p_{t}(x)$, for each $x \in \sigma$. If the sets $\tau$ and $\sigma$ are immune, we can reduce the problem to the finite case by $C 5$.

Proposition C8. Let $\mathscr{P}=\langle\tau, \sigma, p\rangle$ be an isolic $\omega$-STT family and let $t, t^{\prime} \in \tau, t \neq t^{\prime}$. Then the equation

$$
p_{t}(x)=p_{r^{\prime}}(x), \quad \text { for } x \in \sigma,
$$


has at most one solution. Moreover, given $t$ and $t^{\prime}$ we can (a) effectively decide whether (I) has a solution, and (b) compute the solution, if there is one.

Proof. Assume the hypothesis. Suppose (I) had two distinct solutions in $\sigma$, say $x_{1}$ and $x_{2}$; put $y_{1}=p\left(t, x_{1}\right), y_{2}=p\left(t, x_{2}\right)$; then $y_{1} \neq y_{2}$, and since $\mathscr{P}$ is STT, we conclude $t=t^{\prime}$, contrary to the hypothesis. Hence (I) has at most one solution in $\sigma$. If the set $\sigma$ is finite, we can compute $p(t, x), p\left(t^{\prime}, x\right)$ for all $x \in \sigma$ and thereby solve the problem. Now suppose that $\sigma$ is infinite, then so is $\tau$. We may assume an element $a \in \sigma$ as known. From $a$ we compute $b=p(t, a)$, $b^{\prime}=p\left(t^{\prime}, a\right)$. If $b=b^{\prime}$ we have already found the unique solution of (I), namely, $x=a$. Henceforth we assume $b \neq b^{\prime}$, i.e., $p(t, a) \neq b^{\prime}$. According to C6 we can compute the unique $t^{\prime \prime} \in \tau$ such that

$$
p\left(t^{\prime \prime}, a\right)=b^{\prime} \text { and } p\left(t^{\prime \prime}, x\right) \neq p(t, x), \quad \text { for all } x \in \sigma .
$$

By C5 we can construct finite sets $\tau^{*}, \sigma^{*}$ such that $a \in \sigma^{*}, t, t^{\prime}, t^{\prime \prime} \in \tau^{*}$ and for $p^{*}=p \mid \tau^{*} \times \sigma^{*}, \mathscr{P}^{*}=\left\langle\tau^{*}, \sigma^{*}, p^{*}\right\rangle$ is an $\omega$-STT family. We claim that

(I) has a solution in $\sigma \Leftrightarrow$ (I) has a solution in $\sigma^{*}$.

If we can establish (2) we are done, for (2) reduces the problem to the solvability of (I) in the finite set $\sigma^{*}$. Only the conditional from the left to the right requires a proof. Assume that (I) has a solution in $\sigma$, say $x=\bar{x}$. If $t^{\prime \prime}$ were equal to $t^{\prime}$, (I) would have no solution in $\sigma$ by (1); hence $t^{\prime \prime} \neq t^{\prime}$. Suppose that $\bar{x} \in \sigma-\sigma^{*}$. Since $\bar{x}$ is the only solution of (I) in $\sigma$, we conclude that $p(t, x) \neq p\left(t^{\prime}, x\right)$, for $x \in \sigma^{*}$. Using (1) and the fact that $p^{*}=p \mid \tau^{*} \times \sigma^{*}$, we obtain

$$
\begin{aligned}
& p^{*}\left(t^{\prime}, a\right)=b^{\prime} \quad \text { and } \quad p^{*}\left(t^{\prime}, x\right) \neq p^{*}(t, x), \quad \text { for } x \in \sigma^{*} \text {, } \\
& p^{*}\left(t^{\prime \prime}, a\right)=b^{\prime} \text { and } p^{*}\left(t^{\prime \prime}, x\right) \neq p^{*}(t, x), \text { for } x \in \sigma^{*} \text {. }
\end{aligned}
$$

Then an application of C6 to the finite $\omega$-STT family $\mathscr{P}^{*}$ would yield $t^{\prime}=t^{\prime \prime}$ which is false. Thus $\bar{x} \in \sigma^{*}$ and (I) has a solution in $\sigma^{*}$.

\section{The main result}

Proposition C9. With every isolic $\omega$-STT family of degree $N$ we can associate an isolic affine $\omega$-plane of order $N$.

Proof. Let $\mathscr{P}=\langle\tau, \sigma, p\rangle$ be an isolic $\omega$-STT family of degree $N$. We may assume without loss of generality that $\tau \subset\left\{5^{y} \in \varepsilon \mid y>0\right\}$, for if not, we can according to R3 replace $\tau$ by $\tau^{*}=\left\{5^{z+1} \in \varepsilon \mid z \in \tau\right\}$, since $\tau \simeq \tau^{*}$. The set $\sigma$ is recursively equivalent to some set which does not contain 0 . By $\mathrm{C} 1$ we may therefore assume without loss of generality that $0 \notin \sigma$. Define an incidence 
system $\Gamma=\langle\delta, \nu$, inc. $\rangle$ as follows: $\delta=\left\{2^{x} 3^{y} \in \varepsilon \mid x, y \in \sigma\right\}, \nu=\lambda \cup \mu \cup \tau$, where $\lambda=\left\{2^{x} \in \varepsilon \mid x \in \sigma\right\}, \mu=\left\{3^{y} \in \varepsilon \mid y \in \sigma\right\}$; the inc. relation is such that for $x, y, u, v \in \sigma, t \in \tau$,

$$
\begin{aligned}
& 2^{x} 3^{y} \text { inc. } 2^{u} \text { if and only if } x=u, \\
& 2^{x} 3^{y} \text { inc. } 3^{v} \text { if and only if } y=v, \\
& 2^{x} 3^{y} \text { inc. } t \text { if and only if } p_{t}(x)=y \text {. }
\end{aligned}
$$

The elements of $\delta$ are called points; those of $\nu$ lines. It follows from the definition of $\Gamma$ that

(1) any two distinct points $q$ and $r$ of $\Gamma$ lie on exactly one line, say $L_{\Gamma}(q, r)$,

(2) calling two lines of $\Gamma$ parallel if they are identical or have no point in common, (1) implies that any two nonparallel lines $u$ and $v$ of $\Gamma$ have exactly one point in common, say $P_{\mathrm{r}}(u, v)$,

(3) for any point $r$ and any line $l$ of $\Gamma$, there is exactly one line of $\Gamma$ passing through $r$ and parallel to $l$, say $Q_{\mathrm{r}}(r, l)$,

(4) $\Gamma$ has three noncollinear points, for if $a$ and $b$ are distinct elements of $\sigma$, the points $2^{a} 3^{a}, 2^{a} 3^{b}, 2^{b} 3^{b}$ are noncollinear.

We now prove that $L_{\Gamma}, P_{\Gamma}, Q_{\Gamma}$ have partial recursive extensions.

$\operatorname{Re} L_{\mathrm{r}}$. Let the points $q=2^{a} 3^{b}, r=2^{c} 3^{d}$ be given, where $q \neq r$; hence $a \neq c$ or $b \neq d$. Then

$$
L_{r}(q, r)=\left\{\begin{array}{l}
2^{a}, \text { if } a=c \& b \neq d, \\
3^{b}, \text { if } a \neq c \& b=d, \\
\text { unique } t \in \tau \text { such that } p_{t}(a)=b, p_{t}(c)=d, \text { if } a \neq c \& b \neq d .
\end{array}\right.
$$

Given $q$ and $r$ we can effectively decide which clause applies. Using condition (2) of D2 we conclude that $L_{\Gamma}(q, r)$ can be computed from $q$ and $r$.

$\operatorname{Re} P_{r}$. Let the nonparallel lines $u$ and $v$ of $\Gamma$ be given. We may restrict our attention to the following four cases.

(I) $u \in \lambda, v \in \mu$, say $u=2^{a}, v=3^{b}$. Then $P_{\mathrm{I}}(u, v)=2^{a} 3^{b}$.

(II) $u \in \lambda, v \in \tau$, say $u=2^{a}$. Then $P_{\Gamma}(u, v)=2^{a} 3^{p(v, a)}$.

(III) $u \in \mu, v \in \tau$, say $u=3^{c}$. Then $P_{\Gamma}(u, v)=2^{a} 3^{c}$, where $a$ is the solution in $\sigma$ of $p_{v}(x)=c$.

(IV) $u, v \in \tau$. Note that for $x, y \in \sigma$,

$$
2^{x} 3^{y} \text { inc. } u \Leftrightarrow p_{u}(x)=y ; 2^{x} 3^{y} \text { inc. } v \Leftrightarrow p_{v}(x)=y .
$$

Given $u$ and $v$ we can effectively decide which of the four cases holds, since $\lambda$, $\mu, \tau$ are separable. In the first two cases $\operatorname{Pr}(u, v)$ can clearly be computed 
from $u$ and $v$, and in Case (III) it can be computed by C7. In Case (IV) there are $x, y \in \sigma$ such that $p_{u}(x)=y=p_{v}(x)$, since $u$ and $v$ are not parallel; we can then effectively solve $p_{u}(x)=p_{v}(x)$ by C8. Let $x^{\prime}$ be the solution and $y^{\prime}=p_{u}\left(x^{\prime}\right)$. Thus $P_{\Gamma}(u, v)=2^{x^{\prime}} 3^{y^{\prime}}$ can be computed.

$\operatorname{Re} Q_{\Gamma}$. Let a point $r$ and a line $l$ of $\Gamma$ be given, say $r=2^{a} 3^{b}$. We distinguish three cases.

(I) $l \in \lambda$, say $l=2^{c}$. Then $Q_{\Gamma}(r, l)=2^{a}$.

(II) $l \in \mu$, say $l=3^{d}$. Then $Q_{\Gamma}(r, l)=3^{b}$.

(III) $l \in \tau$. Let $Q_{\Gamma}(r, l)=t$. Every line in $\lambda \cup \mu$ intersects every line in $\tau$; hence $t \notin \lambda \cup \mu$ and $t \in \tau$. Also,

$$
2^{a} 3^{b} \text { inc. } l \Leftrightarrow p_{l}(a)=b,
$$

where we can test whether $p_{i}(a)=b$; if this is the case, $r$ inc. $l$ and $t=l$. Now suppose $p_{l}(a) \neq b$, that is, not ( $r$ inc. $l$ ). Then the conditions on $t$ are

(i) $r$ inc. $t$, that is, $p_{t}(a)=b$,

(ii) $l$ and $t$ have no common point, that is, $p_{l}(x) \neq p_{t}(x)$, for all $x \in \sigma$. According to C6 there is exactly one $t \in \tau$ which satisfies (i) and (ii) and it can be computed from $a, b$ and $l$.

We have proved that $\Gamma=\langle\delta, \nu$, inc. $\rangle$ is an affine $\omega$-plane. Since $\lambda=$ $\left\{2^{x} \in \varepsilon \mid x \in \sigma\right\}$, we see that $\operatorname{Req} \lambda=\operatorname{Req} \sigma=N$. Then $o(\Gamma)=N$ by (d).

Definition D7. Let $\Gamma=\langle\delta, \nu$, inc. $\rangle$ be an affine $\omega$-plane of order $N$. Then $\Gamma$ is normal, if there is a set $\sigma$ such that

(1) $0 \notin \sigma$ and $\operatorname{Req} \sigma=N$,

(2) $\Gamma$ has two distinct parallel sets $\lambda$ and $\mu$ such that

$$
\begin{gathered}
\lambda=\left\{2^{x} \in \varepsilon \mid x \in \sigma\right\}, \quad \mu=\left\{3^{y} \in \varepsilon \mid y \in \sigma\right\}, \\
l \in \nu-(\lambda \cup \mu) \Rightarrow(\exists z)\left[z>0 \& l=5^{z}\right],
\end{gathered}
$$

(3) $\delta=\left\{2^{x} 3^{y} \in \varepsilon \mid x, y \in \sigma\right\}$,

(4) $l \cap m=l m$, for $l \in \lambda, m \in \mu$.

Proposition C10. With every affine $\omega$-plane of order $N$ we can associate a normal affine $\omega$-plane of order $N$.

Proof. Let $\Gamma=\langle\delta, \nu$, inc. $\rangle$ be an affine $\omega$-plane of order $N$. Let $\sigma \in N$ and $0 \notin \sigma$. We know by (d) that every parallel set of $\Gamma$ has RET $N$. Let $\lambda_{0}$ and $\mu_{0}$ be two distinct parallel sets of $\Gamma, l_{0} \in \lambda_{0}, m_{0} \in \mu_{0}$; from now on we keep $l_{0}$ and $m_{0}$ fixed. Since $\operatorname{Req} \lambda_{0}=\operatorname{Req} \mu_{0}=N$, there exist functions $l_{x}$ and $m_{x}$ with partial recursive one-to-one extensions such that $\delta l=\delta m=\sigma, \rho l=\lambda_{0}, \rho m=$ $\mu_{0}$. Thus $\lambda_{0}=l(\sigma), \mu_{0}=m(\sigma)$. Put 


$$
\begin{gathered}
\delta h=\varepsilon, \quad h(z)=5^{z+1}, \quad \tau=\nu-\left(\lambda_{0} \cup \mu_{0}\right), \quad \bar{\tau}=h(\tau), \\
\bar{\lambda}=\left\{2^{x} \in \varepsilon \mid x \in \sigma\right\}, \quad \bar{\mu}=\left\{3^{y} \in \varepsilon \mid y \in \sigma\right\} .
\end{gathered}
$$

We now define an incidence system $\bar{\Gamma}=\langle\bar{\delta}, \bar{\nu}, \overline{\text { inc. }}\rangle$ by:

$$
\bar{\delta}=\left\{2^{x} 3^{y} \in \varepsilon \mid x, y \in \sigma\right\}, \quad \bar{\nu}=\bar{\lambda} \cup \bar{\mu} \cup \bar{\tau},
$$

and for $x, y, u, v \in \sigma, \bar{t} \in \bar{\tau}$,

$2^{x} 3^{y} \overline{\text { inc. }} 2^{u}$ if and only if $x=u$,

$2^{x} 3^{y} \overline{\text { inc. }} 3^{v}$ if and only if $y=v$

$2^{x} 3^{y} \overline{\text { inc. }} \bar{t}$ if and only if $P_{\Gamma}[l(x), m(y)]$ inc. $h^{-1}(\bar{t})$.

Define a function $c$ as follows:

$$
\begin{array}{ll}
\delta c=\delta \cup \nu, c(t)=h(t), & \text { for } t \in \tau, \\
c l(x)=2^{x}, c m(y)=3^{y}, & \text { for } x, y \in \sigma, \\
c P_{\Gamma}[l(x), m(y)]=2^{x} 3^{y}, & \text { for } x, y \in \sigma .
\end{array}
$$

Then $c$ is a one-to-one function from $\delta \cup \nu$ onto $\bar{\delta} \cup \bar{\nu}$ with $c(\delta)=\bar{\delta}$,

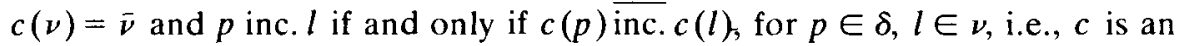
isomorphism from $\Gamma$ onto $\bar{\Gamma}$. Thus, since $\Gamma$ is an affine plane, so is the incidence system $\bar{\Gamma}$. We claim that the three sets $\lambda_{0}, \mu_{0}, \tau$ are separable. For let $p_{0}=P_{\Gamma}\left(l_{0}, m_{0}\right)$ and $t_{0}$ any line of $\tau$ through $p_{0}$. Then we have for $l \in \nu$,

$$
\begin{gathered}
l \in \lambda_{0} \Leftrightarrow Q_{\Gamma}\left(p_{0}, l\right)=l_{0}, \quad l \in \mu_{0} \Leftrightarrow Q_{\Gamma}\left(p_{0}, l\right)=m_{0}, \\
l \in \tau \Leftrightarrow Q_{\Gamma}\left(p_{0}, l\right) \notin\left(l_{0}, m_{0}\right) .
\end{gathered}
$$

Since $Q_{\Gamma}$ has a partial recursive extension, we conclude that the three sets $\lambda_{0}$, $\mu_{0}, \tau$ are separable. Thus the function $c$ has a partial recursive extension. Using the fact that the sets $\bar{\lambda}, \bar{\mu}, \bar{\tau}$ are separable we see that the function $c^{-1}$ also has a partial recursive extension. It follows that the isomorphism $c$ from $\Gamma$ onto $\vec{\Gamma}$ has a partial recursive one-to-one extension; thus, since $\Gamma$ is an affine $\omega$-plane, so is $\bar{\Gamma}$. Let $l \in \nu, \bar{l}=c(l)$; then $c\left(\delta_{l}\right)=\bar{\delta} \bar{i}$. Hence Req $\delta_{l}=\operatorname{Req} \bar{\delta}_{\bar{l}}$, that is, $o(\Gamma)=o(\bar{\Gamma})$. Finally, $\Gamma$ is normal by its definition.

Proposition C11. With every isolic affine $\omega$-plane of order $N$ we can associate an isolic $\omega$-STT family of degree $N$.

Proof. Let $\Gamma=\langle\delta, \nu$,inc. $\rangle$ be an isolic affine $\omega$-plane of order $N$. In view of C10 we may assume that $\Gamma$ is normal. Let $\sigma$ be a set in $N$ and $\lambda, \mu$ two 
distinct parallel sets of $\Gamma$ such that the four conditions of D7 are satisfied. Let $\tau=\nu-(\lambda \cup \mu)$. For every $t \in \tau, x \in \sigma$, we have $2^{x} \in \lambda$, so that $t \cap 2^{x}$ is a well-defined point of $\Gamma$. Define the function $p$ by: $\delta p=\tau \times \sigma$ and for $(t, x) \in \delta p$,

$$
p(t, x)=\text { unique } y \in \sigma \text { such that } t \cap 2^{x}=2^{x} 3^{y} .
$$

For each $t \in \tau, p(t, x)=p_{t}(x)$ is a function from $\sigma$ into $\sigma$. Let $\mathscr{P}=$ $\left\{p_{t} \in \sigma^{\sigma} \mid t \in \tau\right\}$. We prove that $\mathscr{P}$ is an $\omega$-STT family on $\sigma$ by showing:

(1) for each $t \in \tau, p_{t}$ is a permutation of $\sigma$,

(2) $p(t, x)$ has a partial recursive extension,

(3) given $(a, c),(b, d) \in(\sigma \times \sigma)^{-}$, there is a unique $t \in \tau$ with $p_{t}(\dot{a})=b$, $p_{t}(c)=d$ and this $t$ can be computed from $a, b, c, d$.

Re (1). Let $t \in \tau$. To show that $p_{t}$ is one-to-one, assume $a, b \in \sigma$ and $a \neq b$. Then $a \neq b$ implies that the points $q=t \cap 2^{a}=2^{a} 3^{p(t a)}$ and $r=t \cap 2^{b}=$ $2^{b} 3^{p(t, b)}$ are distinct so that $t=q \cdot r$. If $p(t, a)$ were equal to $p(t, b)$, say $p(t, a)=p(t, b)=m$, the line $t$ would have two distinct points in common with the line $3^{m}$, namely, $2^{a} 3^{m}$ and $2^{b} 3^{m}$; then $t=3^{m}$ and $t \in \mu$, contrary to the fact that $t \in \tau$. Hence $p_{t}(a) \neq p_{t}(b)$. To show that $p_{t}(\sigma)=\sigma$, assume $s \in \sigma$. The line $t$ intersects the line $3^{s} \in \mu$, say in $2^{r} 3^{s}$; then $t \cap 2^{r}=2^{r} 3^{s}$ and $p_{t}(r)=s$. We have proved that $p_{t}$ is a permutation of $\sigma$.

$\operatorname{Re}(2)$. Given $(t, x) \in \tau \times \sigma$, we see that $p(t, x)$ is the unique $y$ such that $t \cap 2^{x}=2^{x} 3^{y}$, that is,

$$
p(t, x)=\text { unique } y \text { such that } P_{\mathrm{r}}\left(t, 2^{x}\right)=2^{x} 3^{y} .
$$

Since $P_{r}$ has a partial recursive extension so has $p(t, x)$.

$\operatorname{Re}(3)$. Let $(a, c),(b, d) \in(\sigma \times \sigma)^{-}$be given. Then

$$
\begin{aligned}
t \in \tau \& p(t, a)=b \& p(t, c)=d & \Leftrightarrow t \in \tau \& t \cap 2^{a} \text { inc. } 3^{b} \& t \cap 2^{c} \text { inc. } 3^{d} \\
& \Leftrightarrow t \in \tau \& 2^{a} 3^{b} \text { inc. } t \& 2^{c} 3^{d} \text { inc. } t \\
& \Leftrightarrow t \in \tau \& t=L_{\Gamma}\left(2^{a} 3^{b}, 2^{c} 3^{d}\right), \text { since } a \neq c, \\
& \Leftrightarrow L_{\Gamma}\left(2^{a} 3^{b}, 2^{c} 3^{d}\right) \in \tau .
\end{aligned}
$$

Put $n=L_{\mathrm{r}}\left(2^{a} 3^{b}, 2^{c} 3^{d}\right)$; then $n$ is well-defined, $n$ has the point $2^{a} 3^{b}$ in common with $2^{a}$ and the point $2^{c} 3^{d}$ with $2^{c}$. However, $a \neq c$ so that $n$ intersects two different lines in $\lambda$, hence $n \notin \lambda$; similarly we see that $n \notin \mu$. Thus $n \in \tau$. Moreover, since $L_{\mathrm{I}}$ has a partial recursive extension, $n$ can be computed from $a, b, c, d$. We have proved that $\mathscr{P}$ is an $\omega$-STT family on the set $\sigma$, where Req $\sigma=N$.

THEOREM. There exists an isolic affine $\omega$-plane of order $N$ if and only if there exists an isolic $\omega$-STT family of degree $N$. 
Proof. C9 and C11.

Proposition C12. There are exactly $c=2^{\kappa_{0}}$ isolic $\omega$-STT families. Among these there are exactly $c$ immune ones.

Proof. D2 implies that there are at most $c \omega$-STT families; hence it suffices to show that there are at least $c$ immune $\omega$-STT families. According to (e) there are $c$ immune affine $\omega$-planes. Denote the continuum by $\mathbb{C}$. Let $\left\{\Gamma_{\lambda} \mid \lambda \in \mathbb{C}\right\}$ be the family of all immune affine $\omega$-planes, where the mapping $\lambda \rightarrow \Gamma_{\lambda}$ is one-to-one. Put $N_{\lambda}=o(\Gamma \lambda)$, for $\lambda \in \mathbb{C}$. With each $\lambda \in \mathbb{C}$ we associate a line $l_{\lambda}$ of $\Gamma_{\lambda}$ and the set $\delta_{\lambda}$ of all points on $l_{\lambda}$. Thus $N_{\lambda}=\operatorname{Req} \delta_{\lambda}$, for $\lambda \in \mathbb{C}$. An infinite isol contains only $\boldsymbol{N}_{0}$ sets, so that $\left\{N_{\lambda} \mid \lambda \in \mathbb{C}\right\}$ is a collection of $c$ infinite isols. For each $\lambda \in \mathbb{C}$ there is an immune affine $\omega$-plane of order $N_{\lambda}$, hence by the Theorem also an immune $\omega$-STT family of degree $N_{\lambda}$. Thus, since $\left\{N_{\lambda} \mid \lambda \in \mathbb{C}\right\}$ has cardinality $c$, there are at least $c$ immune $\omega$-STT families.

\section{Concluding remarks}

R5. Let $\mathscr{P}_{1}=\left\langle\tau_{1}, \sigma_{1}, p_{1}\right\rangle$ and $\mathscr{P}_{2}=\left\langle\tau_{2}, \sigma_{2}, p_{2}\right\rangle$ be two $\omega$-STT families. Then we define

$$
\begin{aligned}
& \mathscr{P}_{1} \leqq \mathscr{P}_{2} \text { as: } \tau_{1} \subset \tau_{2} \& \sigma_{1} \subset \sigma_{2} \& p_{1}=p_{2} \mid \tau_{1} \times \sigma_{1} \\
& \mathscr{P}_{1}<\mathscr{P}_{2} \text { as: } \mathscr{P}_{1} \leqq \mathscr{P}_{2} \& \mathscr{P}_{1} \neq \mathscr{P}_{2} .
\end{aligned}
$$

Let $\left\langle\mathscr{P}_{n}\right\rangle$ be an infinite sequence of $\omega$-STT families, where $\mathscr{P}_{n}=\left\langle\tau_{n}, \sigma_{n}, p_{n}\right\rangle$, for $n \in \varepsilon$. Then $\left\langle\mathscr{P}_{n}\right\rangle$ is an ascending (strictly ascending) chain, if $\mathscr{P}_{n} \leqq \mathscr{P}_{n+1}$ (respectively, $\mathscr{P}_{n}<\mathscr{P}_{n+1}$ ), for $n \in \varepsilon$. If $\left\langle\mathscr{P}_{n}\right\rangle$ is an ascending chain, we define $\cup \mathscr{P}_{n}$ as the system $\mathscr{P}=\langle\tau, \sigma, p\rangle$ such that

$$
\tau=\bigcup_{n \in \varepsilon} \tau_{n}, \quad \sigma=\bigcup_{n \in \varepsilon} \sigma_{n}, \quad p=\bigcup_{n \in \varepsilon} p_{n},
$$

where $p$ and $p_{n}$ are interpreted as collections of ordered pairs. Note that $\mathrm{C5}$ implies that an immune $\omega$-STT family $\mathscr{P}$ can always be expressed in the form $\mathscr{P}=\cup \mathscr{P}_{n}$, where $\left(\mathscr{P}_{n}\right\rangle$ is a strictly ascending chain of finite $\omega$-STT families. A similar remark can be made about an immune affine (or projective) $\omega$-plane.

R6. An $\omega$-group is an ordered pair $\langle\alpha, o\rangle$, where (i) $\alpha \subset \varepsilon$, (ii) $o$ is a group operation on $\alpha \times \alpha$ which has a partial recursive extension, and (iii) the function $g(x)=x^{-1}$, for $x \in \alpha$, has a partial recursive extension. These $\omega$-groups were introduced by M. J. Hassett (1969) and also studied by C. H. Applebaum (1971). Now consider an $\omega$-STT family of permutations $\mathscr{P}=$ $\langle\tau, \sigma, p\rangle$. In case $\mathscr{P}$ is a group, we define for $u, v \in \tau$, 


$$
u \circ v=\text { unique } t \in \tau \text { such that } p_{t}=p_{u} p_{v} \text {. }
$$

We like to point out that if $\mathscr{P}$ is a group, then $\langle\tau, o\rangle$ is an $\omega$-group. For let $a, c \in \sigma$, where $a \neq c$, and let $u, v \in \tau$. Since $p(t, x)$ has a partial recursive extension, we can compute the numbers $r=p_{u} p_{v}(a)$ and $s=p_{u} p_{v}(c)$. However, $a \neq c$ implies $r \neq s$ and $\tau$ contains exactly one $t$ such that $p_{t}(a)=r$ and $p_{1}(c)=s$; hence this $t$ equals $u \circ v$. It follows that the group operation $o$ has a partial recursive extension. Let $g(t)=t^{-1}$, for $t \in \tau$. Given $t \in \tau$ we can compute the numbers $b=p_{t}(a)$ and $d=p_{t}(c)$. However, $a \neq c$ implies $b \neq d$ and $\tau$ contains exactly one $t^{\prime}$ such that $p_{t^{\prime}}(b)=a$ and $p_{r^{\prime}}(d)=c$; hence this $t^{\prime}$ equals $g(t)$. We conclude that the function $g(t)$ also has a partial recursive extension so that $\langle\tau, o\rangle$ is an $\omega$-group.

\section{Acknowledgment}

We wish to thank Dr A. Silverstein for her careful reading of the manuscript and her criticisms.

\section{REFERENCES}

C. H. Applebaum (1971), ' $\omega$-Homomorphisms and $\omega$-groups', Journal of Symbolic Logic 36, 55-65.

J. C. E. Dekker and J. Myhill (1960), 'Recursive equivalence types', University of Califormia Publications in Mathematics (N. S) 3, 67-214.

J. C. E. Dekker (1977), 'Planos afines con operaciones recursivas', Ciencia y Tecnologiá, Revista de la Universidad de Costa Rica, 1, 13-29. See also abstract, Notices American Mathematical Society 23 (1976), A-597.

P. Dembowski (1968), Finite geometries (Springer-Verlag, New York).

M. Hall (1943), 'Projective planes', Transactions of the American Mathematical Society 54, 229-277.

M. J. Hassett (1969), 'Recursive equivalence types and groups', Journal of Symbolic Logic 34, 13-20.

Rutgers, The State University,

New Brunswick, New Jersey 08903, U.S.A. 